Orginal article

\title{
Mazandran based radiologists' attitudes to obtaining patients' consent prior to invasive imaging procedures, 2010
}

\author{
Benyamin Mohseni Saravi ${ }^{1}$, Gholam Reza Fallah Mohammadi ${ }^{2}$, Mahboube Yahghobian ${ }^{3, *}$, Hasan Siamian ${ }^{4}$
}

(Received: 17 Aug 2014; Accepted: 16 Nov 2014)

\begin{abstract}
Background and Purpose: In some patients, invasive radiology procedures by using anesthetics and contrast may lead to certain reaction. Therefore, consent must be obtained from patients. At the moment, some radiology centers obtain the consent from the patient in some of invasive radiology procedures in Iran. Due to the importance of consent and its position in patients' ethics, and because of lack of national order, and by considering the effect of individual attitude on the execution of each process; therefore, in this study, the attitude of radiologists as supervisors of the executive policies of a radiology center has been investigated.

Methods: This cross-sectional descriptive study was done in Mazandran province in Iran. The study population consists of all radiologists (66 radiologists) from 83 radiology centers. A questionnaire with alpha 0.8 designed at 5 axes and self-administration was used. The data were analyzed by descriptive analysis indices and using SPSS software.

Results: from 83 radiology centers with 66 radiologists, only 51(77\%) filled in the study questionnaire. The number of images taken per day was 45 on average and the invasive images were $2 \pm 2$.The participants under study had positive attitudes towards obtaining consent form.
\end{abstract}

Conclusion: Considering the positive attitudes of participants under study, it seems that the radiologists would agree to get consent form of the patients undergoing the invasive procedure of imaging.

Keywords: Consent, Attitude, Radiology, Patient rights

\section{Introduction}

The element of consent is one of the critical issues in the area of medical treatment today. It is well known that the patient must give valid consent for medical treatment; and they are entitled to refuse treatment even if the question treatment saves his or her life. No doubt, this raises many ethical debates and falls at the heart of medical law today. The earliest expression of this fundamental principle, based on autonomy, is found in the Nuremberg Code of 1947 (1). The MCI guidelines are related to operations and do not cover other treatments. For other treatments, the following may be known as the general guidelines: for routine types of treatment, implied consent would suffice, for detailed types of treatment, ideally expressed oral consent may be needed and finally, for

\footnotetext{
${ }^{1}$ Medical Records MSc, Mazandran University of Medical Sciences, Mazandran, Sari, Iran.

${ }^{2}$ Department of Physics and Engineering Medicine, Tehran University of Medical Sciences. Tehran, Iran.

${ }^{3, *}$ Corresponding author: Department of Nursing Management, Mazandran University of Medical Sciences. Traditional and Complementary Medicine Research Centre, Mazandran University of Medical Sciences, Sari, Iran.

Nasibeh Nursing and Midwifery School, Mazandran University of Medical Sciences, Sari, Iran. E-mail: yaghobian2005@yahoo.com.

${ }^{4}$ Department of Health Information Technology, School of Allied Medical Sciences, Mazandran University of Medical Sciences, Sari, Mazandran, Iran.
} 
complex types of treatment, written expressed consent is required (2). Obtaining consent from obtained consent for the treatment and procedures (3). Today, obtaining the consent from patients is necessary (4) . Getting consent for medical care is a legal force; consent forms are written behind the reception forms (3). Consent is a process through which patients or their surrogate decision makers understand and agree with the medical procedures. Consent in the form of written and oral is accepted. Thus, it is necessary to emphasize and highlight the importance of obtaining consent. (5). Doctors need to touch their patients, whether for simple examination or for more invasive interventions. Although oral consent is adequate in English law for any of these procedures, there is an understandably low threshold for obtaining written consent (6). Some researchers mentioned that in outpatient treatment, there is no need to sign a consent form and preserve it (7). The studies' results showed that in Texas, physicians are required to get written consent for procedures or treatments indicated by Texas Medical Disclosure Panel (TMPD). The TMDP has created two separate lists of those medical treatments and surgical procedures that do and do not require disclosure. As is the case with all patient interactions, the consent process should be documented in the medical record. Anesthesiologists and radiologists often make a mistake relying on the primary or attending physician to handle the consent process on their behalf, making an assumption that the primary physician has adequately addressed the risks associated with the intended anesthesia or radiological procedure. The only person who is truly qualified to respond to a patient's questions satisfactorily is the physician specialist who is to perform the procedure. Consent is no longer limited to surgical, invasive, or diagnostic procedures. Written consent also should be obtained for drugs that involve potentially significant complications or side effects (8). There are many instances where consent is necessary in radiology practice: before administering intravenous contrast, before interventional procedures of all types, and before administering anesthesia. In all such situations, it is necessary to follow proper procedure regarding consent: the consent form itself must be comprehensive and cover all issues of importance including major and common complications based on an explanation given to the patient and his/her relatives/friends in their own language, the signature or thumb impression [finger print] of the patient must be taken, a relative or attendant must endorse this consent at the same time, and no changes must be made to the consent form thereafter.(9). It is evident that lack of consent against any claims brought by patients may be condemnations on hospitals. Consent was not dictated by the patient's rights, treatment providers should be accountable to patients.

Today para -clinical services such as radiology have many applications in the diagnosis and treatment of the diseases. In some case, it is done by anesthetic and introduction of contrast which may lead to a reaction from the patients. Therefore, obtaining consent from the patient is mandatory (10). Consent is a must because in case of the patient complaining about attending is helpful. There is no difference between the outpatient and inpatient or the specialty of the care giver, in such a manner that when the patient complains, it would be investigated. Nowadays physician-patient relationship has deteriorated due to socio-economic problems, as well as high specialization in medical practice (11). In the UK, the records demonstrate 1000 registered complaints out of six million inpatients and 19 million out-patients in 1978 (12). Farhady documented 8 (1.4\%) malpractice cases reported to the medical council related to radiologists (13).

In Iran, only some imaging centers obtain consent from the patients before the invasion but it's still a mystery whether the confusion caused by lack of consent in other centers can be due to lack of knowledge or the attitudes of their leaders. 
Obviously adequate attitude also can prevent the occurrence of errors and legal issues. Regarding the above-mentioned cases about the importance of consent and its position in the legal system of the patient, and the absence of national guidelines in this regard, the ministry officials insist on the new patients' bill of rights, since the medical universities support researches about patients' rights, and the unquestionable impact of attitude on performance. In this study, the attitudes of radiologists as factors relating to policy implementation of an imaging center is investigated. The results of this study may provide the starting point for the administrative authorities to formulate the appropriate strategies for the legal interests of the patient.

\section{Materials and Methods}

This cross-sectional descriptive study was done in Mazandran province (northern Iran) in 2011. The study population was 66 radiologists from 83 imaging centers selected by census method. If a radiologist managed two clinic centers at the same time, he/she was excluded from one of them. Questionnaire comprising two separate sections with 13 statements (the radiology center information and the attitude questions) designed based on the objectives of the study written by reviewing the literatures and the new version of patient's Bill of Rights of the Ministry of Health of Iran. This questionnaire has face validity provided by consulting with 6 faculty members' legal advisers some of whom were outside the imaging centers of Mazandran province. It was filled by self-reporting questionnaires. Its reliability was confirmed in a pilot study with 15 questionnaires totally among radiology students and with alpha coefficient as 0.80 . Attitude statements were designed in 5 areas: autonomy $(1,13)$, patient's knowledge $(4,7,10,12)$, the importance of getting consent (6, 9), legal aspects (2, 3, 5, 8), and consent validity (11). Questionnaire was distributed by mail and they were invited by telephone. Medical imaging centers with no interest to participate in this study were excluded from the study. The attitude-based questionnaire includes 13 questions having options as agree 1; neither agree nor disagree 2; and disagree 3, respectively. Agreement over $50 \%$ in any questions in each area was regarded as positive. The data have been analyzed using descriptive statistics \& SPSS software. Complying with the legal issues like confidentiality, presenting the medical imaging center, the physician, or data have been avoided based on separation and then results were reported generally after being analyzed.

\section{Results}

Of 83 radiology centers with 66 radiologists, 51 (77\%) participating in this study responded to all the statements in the questionnaire. The completed questionnaires were considered for final analysis. The activity of medical imaging centers is given in diagram 1 and the affiliation of radiology centers in Mazandran province is presented in diagram 2.

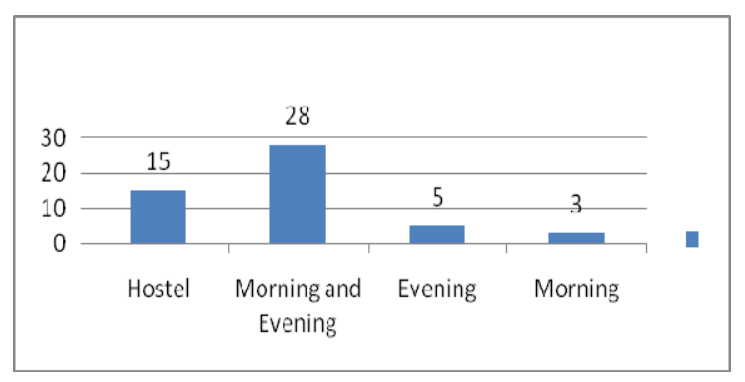

Diagram 1. The activity of medical imaging centers in Mazandran province in 2011

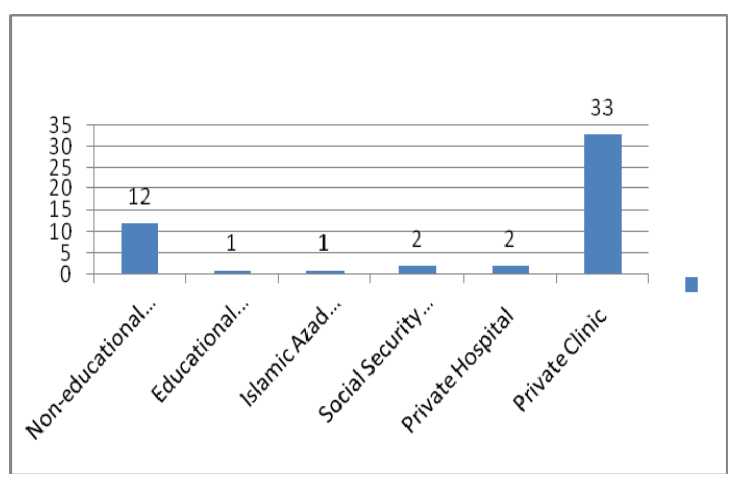

Diagram 2. The affiliation of radiology centers in Mazandran province in 2011 
The results suggested that only 40 centers provided radiology services and 11 centers provided all imaging services. On average, the centers have $6 \pm 3$ employees, the average number is 45 cases of imaging in a day, and the mean number $2 \pm 2$ of invasive imaging was done in a given day. The results of Iranian radiologists 'attitudes towards obtaining consent based on the relevant variation are given in the table 1.

Table 1. Iranian radiologists' attitudes towards obtaining consent from the patients prior to the invasive radiology procedures in 2011

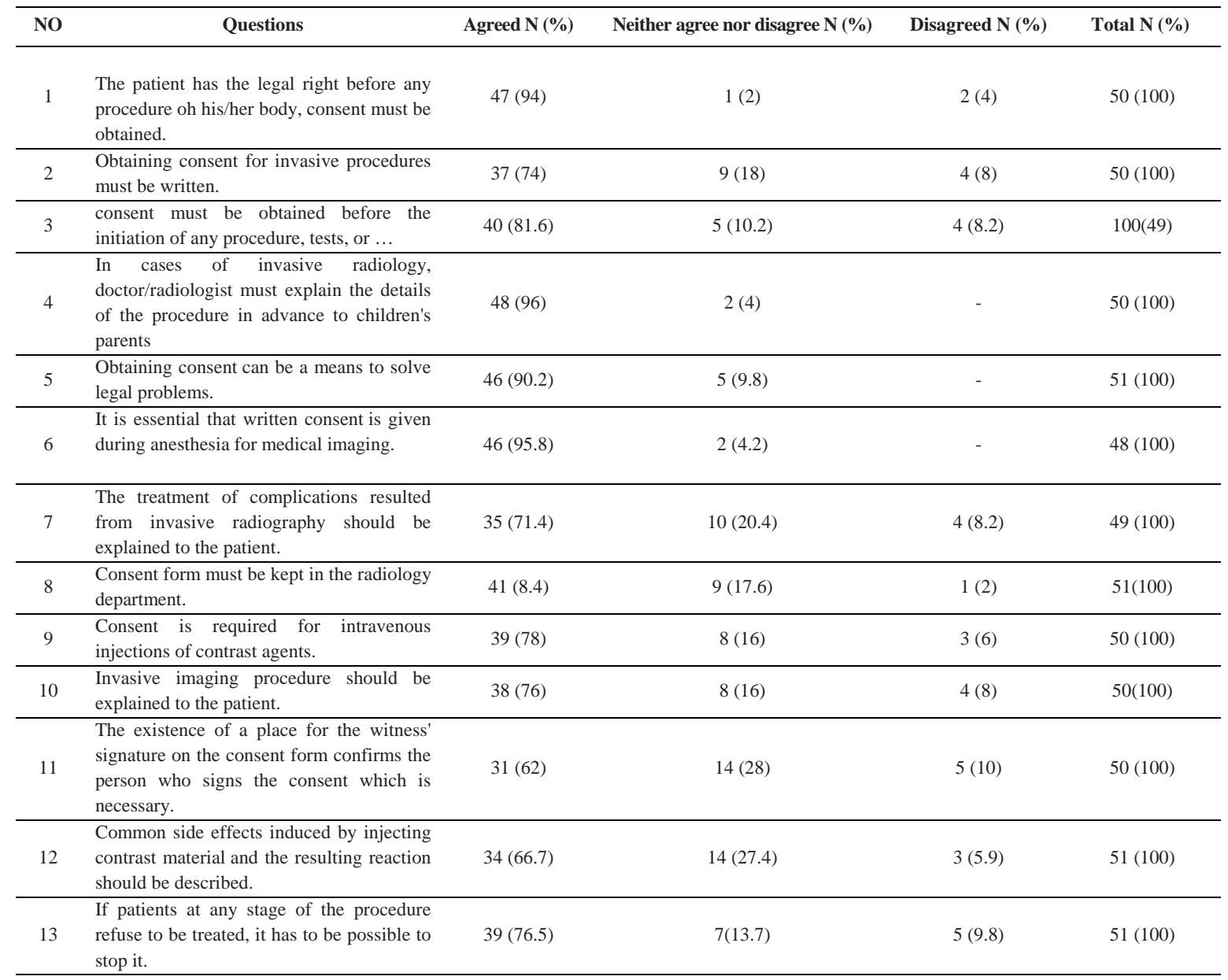

As the results revealed, on average out of 83 radiology centers with 66 radiologists, 51(77\%) participated in this study and filled the questionnaire. Results indicated that only 40 centers provided radiology services and 11 centers supplied all imaging services. Averagely speaking, the centers have $6 \pm 3$ employees, the average number (45) cases of imaging in a day, and the mean number ( $2 \pm 2)$ of invasive imaging in a given day.

As the results showed, on average 2 invasive images were taken per day, and considering the effect of invasion on the patients, consent has to be obtained from the patient and the patients' parents $(1,3,5-6,9-10,12-16)$. In this regard, a national form containing the relevant guidance is necessary. Of course in the present study, in some countries the centers in charge designed an innovative form and obtained consent from the patients and sometimes attached this form to the questionnaire and sent them to us being little informative. It is obvious that their legal validity was not clear. In the anatomy area, the high rate of agreement was noticed among the respondents who agreed with the data given by JammJoom $(14,17)$. 
It emphasizes the obvious significance of the patient's rights regarding self-control for physicians.

In the patient's Bill of Rights, it is clarified that they should respect the patient's rights in terms of freely deciding to receive or reject medical services (2-3). Also they must pay attentions to the patient' rights on giving up the treatment process. Discharge from the hospital based on the patient's decision has been predicted. Data revealed that most of the participants had positive attitude towards knowledge area. It is obvious that giving knowledge on the performance mode, the common complication and the treatment style in case ofcomplication incidence could help the treatment staff in solving the treatment problem of the patients. Amani Sasani in his research emphasizes that the physician is bound to give proper information about the treatment and diagnosis \& the potential side effects. Even giving the necessary instruction to the patients in referring to the physician in case of suspecting the complication is required $(6-7,15,18)$. The data about attitude assessment $\mathrm{n}$ the legal aspects showed that the participants have positive attitude towards obtaining the written consent before the treatment process, the position of consent in solving the legal issues and also keeping the consent form. Of course, in case of making complaints, the written consent is accepted and the oral consent is not valid $(4-7,11)$.

There was also positive attitude towards the significance of consent form on invasive anesthesia and giving necessary exploration about the type of invasion along with imaging. Of course, $27.5 \%$ of the patients have neutral opinion. One of the reasons could be the psychological effects or even the treatment itself on the patient and basically, explaining to the patient seems harming. In return for presenting the explanations, the patients showed different responses such as mental stress. Even sometimes explaining the treatment process is harmful. Explanation to the patient may cause the patient avoiding treatment. In such cases, there are different recommendations. Isfahani in his book recommended that in such cases, explaining to the patients' companions it enough because the patient isn't psychologically ready to hear such explanations (16-19).

The study subjects had positive attitude towards the validity of the consent even obtained from the patient's relative. Kabirzadeh et al. found that $74.6 \%$ of the gained consent lacked validity compared with the standard criteria $(20,17)$. Hajavi reported that despite not being in the legal age, $2.4 \%$ of the patients gave consent and in $3.6 \%$ consent was given by an individual without having any relation with the patients $(1,21)$. It is vivid that validity is integral part of any document, and since the consent form is a type of document between the provider and the receiver; therefore, the legal aspect should be regarded in order to allow to defend in favor of the patients or even the service providing center. It has been clarified that if the patient can't give written consent due to hand problem, obtaining consent should not be avoided. In one case like this, the complaint of the patient similar to such case resulted in the decision be given in favor of the patient $(22,18)$.

\section{Conclusion}

To draw conclusion and based on the present study data, it has been clarified that the study subjects have had positive attitude and it seems that the circumstances are ready in accepting consent from the patients in case of taking invasive imaging. However, all of the actions in favor of the patients' rights are focused by the medical ethical committee and it seems that some time has to be allocated to this part \& take actions to defend patient.

\section{Conflict of interest}

The author(s) declare that they have no competing interests. 


\section{Author's contributions}

All authors participated in writing the scientific proposal, data collection, and writing of the manuscript. All authors read and approved the final manuscript.

\section{Acknowledgments}

All of the radiologists who participated in this study are sincerely appreciated. This article was extracted from Proposal No. 90-76 accepted by Mazandran University of Medical Sciences, Sari, Iran.

\section{References}

1. Nandimath OV. Consent and medical treatment: The legal paradigm in India. Indian j Urolo. 2009; 25(3): 343-347.

2. Medical Council of India. Regulation 7.16 of Medical Council of India (Professional Conduct, Etiquette and Ethics) Regulations. 2002.

3. Hajavi A, Piri Z, Shahmoradi L, Asadi N, Kheradmandi S, Ovaisi $\mathrm{M}$, et al. A survey on completeness of inpatient informed consent forms in the three treatment teaching centers in IRAN University of medical sciences Persian. $\mathrm{J}$ health adm. 2008; 11(32): 55-62 (Persian).

4. Parsapour A, Bagheri A, Larijani MA. Bill of Patients' Rights in Iran. Iranian Journal of Medical Ethics and History of Medicine, 2010. 3 (Special Issue). Iranian Journal of Medical Ethics and History Winter. 2009, 3 (Supplement): 39-47 (Persian).

5. Taghadosinejad F, Sheykh Azadi A, Yaghmaei AA, Rojaei M. A survey on obtaining informed consent from patients admitted to Shariati Hospital (Tehran, Iran). J Med Counc I R Iran. 2008; 26(1):42-9 (Pesian).

6. Elmalik K, Wheeler R. Consent: luck or law? Ann R Coll Surg Engl. 2007; 89(6): 627-630.

7. Amani Sasani R. The Key points in planning a proper consent. Symposium on the legal nature and content of medical consent. 2009 (Persian).

8. Informed Consent: a Process of Communication. avaiable Texas Medical Association. Texas 2014 [cited 2014 September 1, 2014].
9. Consent in radiology practice. Indian $\mathrm{J}$ Radiol Imaging. 2009; 19(1): 21-22.

10. Rahimi S, Khorami F, Mesbahi AR, Saief R, Abtahi M, Davodi L. Consent in the point of viewe of physicians of teaching hospital of Hormozgan University of Medical Sciences. Bandar Abbas(Iran); First national congress on malpractice and medical errors, 24-26 December: Hormozgan University of Medical Sciences. 2008; 167-79 (Persian).

11. Khavanin-zadeh M, Gholipour F. Complaints related to laparoscopic surgery: a survey of the files registered at Tehran legal medicine center. Int $\mathrm{J}$ Hosp Res. 2013; 2(4): 213-216.

12. Poloson G, Gee N. The Essentials of Forensic Medicine 5 th ed. Oxford: Pergamon Press; 1985.

13. Farhadee Y, Golmakani M, Haghighi Z, Pirzadeh R. A Survey of Medical Errors in Tehran 1987-1998. Teb Va Tazkieh. 2005; 55: 21-6.(Persian)

14. Hajavi A, Khoshgam M, Moosavi M. A study of knowledge and considering of consent legal aspect in teaching hospitals of Shahid Beheshti University of Medical Siences; 2008. J Health Adm. 2009; 12(36): 47-52 (Persian).

15. Tavakoli N, Sagheiannejad S, Ajami S, Gheshlaghi F,Abbasi Shirin, Moosavi M. The medical records as a forensic resource. firtst ed. Isfahan: Isfahan University of Medical Sciences publication; 2008.

16. Larijanni B, Jafarri A, Kazemian A, Sadre Hossaini SM. Health care professional and ethical issue. Barye Farda; 2004.

17. Jamjoom AA, White S, Walton SM, Hardman JG, Moppett IK. Anaesthetists' and surgeons' attitudes towards informed consent in the UK: an observational study. BMC medical ethics. 2010; 11(1): 2.

18. Seyed Fatemi SM. Concealed Consent. J Reprod Infertil. 2000; 1(4): 33-37.

19. Esfahani MM. Professional ethics in health. Tehran: Iran University of Medical Sciences; 1992.

20. Kabirzadeh A, Delaram A, Abbasi M, Payesteh S, Taheri M. Rate of consent validity in patients from teaching hospitals in Sari.. J Mazandaran Univ Med Sci. 2009; 18(69): $62-7$.

21. Hajavi A, Piri Z, Shamoradi L, Asadi N, KHeradmandi S, Ovaisi $\mathrm{M}$, et al. A survey on completeness of inpatient informed consent forms in the three treatment teaching centers in IRAN 
University of medical sciences[Persian]. Journal of health administration. 2008; 11(32): 55-6 (Persian).
22. Signature on consent form when the patient's right/left hand is impaired. Indian J Radiol Imaging. 2009; 19(1): 22-23. 